\title{
SLEEP DISORDERS IN CHILDREN WITH EPILEPSY
}

Sleep and behavior disorders in 55 children with epilepsy (mean age 10 years; range 4-16 years) were compared with those in their non-epileptic siblings of the same ages and sex ratio and correlated with epilepsy-specific factors, in a study at Alberta Children's Hospital, University of Calgary, Alberta, Canada. Sleep Behavior Questionnaires (SBQ), Child Behavior Checklists (CBCL), and Quality of Life (QOL) Scores were completed by parents and caregivers. Epilepsy was idiopathic generalized in $8(15 \%)$, symptomatic generalized in $7(13 \%)$, and focal in $40(73 \%)$. Epileptiform discharges in the EEG were present in 51 patients, and in $62 \%$ of 39 with sleep records. Only 5 had nocturnal seizures. Seizures had been controlled for at least 3 months in $53 \%$, and for 1 year in $24 \%$. Treatment was monotherapy in $47 \%,>2$ AEDs in $38 \%$, and no AED in $15 \%$.

Children with epilepsy have significantly longer sleep latency, increased parent-child interaction at night, increased sleep fragmentation, increased parasomnias, and increased daytime drowsiness compared to non-epileptic siblings. They also score significantly higher on behavior subscales (withdrawn, somatic complaints, social problems, thought and attention problems, and aggressive behavior) compared to controls. Higher total sleep disorder scores are correlated with higher scores on CBCL subscales (withdrawn behavior, somatic complaints, social problems, and attention deficits), and significantly lower total QOL scores. Refractory epilepsy (35\%), mental retardation (53\%), and remote symptomatic etiology are risk factors for greater sleep problems with epilepsy. (Wirrell E, Blackman M, Barlow K, et al. Sleep disturbances in children with epilepsy compared with their nearestaged siblings. Dev Med Child Neurol Nov 2005;47:754-759). (Respond: Elaine Wirrell MD, Alberta Children's Hospital, 1820 Richmond Road SW, Calgary, Alberta, Canada T2T 5C7).

COMMENT. Children with epilepsy have increased sleep problems compared to nonepileptic siblings, and sleep disorders are correlated with daytime behavior problems and a poorer quality of life. Sleep patterns are an important question in the evaluation of children with epilepsy.

\section{HEADACHE DISORDERS}

\section{SLEEP DISORDERS IN CHILDREN WITH HEADACHE}

The prevalence of sleep disorders in 64 patients (38 [59\%] males and 26 [41\%] females; average age 10 years) with headache was evaluated in the Pediatric Neurology outpatient clinics, University of Chicago. Compared to 64 matched controls without headache, children with headache had a significantly higher prevalence of excessive daytime sleepiness, narcolepsy, and insomnia $(\mathrm{p}<0.005)$, but the prevalence of sleep apnea, parasomnias, and restlessness was not increased. In 18 patients diagnosed with migraine headaches, the scores for excessive daytime sleepiness and narcolepsy were significantly higher, but those for insomnia, parasomnia, sleep apnea, and restlessness were not different from non-headache controls. Medications did not alter the prevalence of any of the sleeping disorder symptoms $(\mathrm{p}>0.05)$. Headache patients had more frequent problems with 